\title{
African American hair loss - what can be done?
}

\section{William D Yates, MD*}

Hair Restoration Surgeon, Yates Hair Science Group, Chicago, IL, USA

Unfortunately, even beautiful super models like Naomi Campbell, Paris Hilton, Victoria Beckham, Britney Spears and Alexandra Burke appear to suffer from a very common problem called traction alopecia as a result of long-term hair extensions.

Traction Alopecia as seen in the above mentioned models and women from every socioeconomic class occurs when extensions or tight braiding pull on the natural hair causing it to weaken and break. This usually affects the hairline just above the forehead or the sides, where the hair is more fragile and a finer texture. Initially traction alopecia is reversible but prolonged tension will destroy the hair follicles preventing any possible new growth of hair. If the tension or the extensions are removed, it can take between three months to a year for hair to grow back in moderate cases. Some medical treatments such asminoxidil, (Rogaine) can help to re-stimulate the hair follicle to grow in early cases where the hair follicle is not permanently injured. In my opinion, a combination of minoxidil and a hair transplant is the best solution to restore hair in this condition.

Balding and hair loss in general for both men and women continues to be a huge problem throughout the world. It affects 35 million men in the US alone and 21 million women in the US [1]. The main source of this unfortunate event is genetic and at this time cannot be stopped if you are predisposed to the balding gene. There are medications such as finasteride and minoxidil, which can slow the balding process and stabilize hair loss mainly in men. Minoxidil is approved for use in women as well. Traction Alopecia appears to be caused by hair styling practices and can be improved or eliminated by an appropriate timely intervention.

Hair is such an important feature for one's appearance. It frames the face and creates a more youthful and pleasing appearance. Luckily, there are some good options available today with techniques progressively improving to help restore your hairline and a more youthful appearance.

Hair transplantation is the only permanent solution to restore lost hair. The history of hair transplantations began in the United States in 1952. Dr. Norman Orientreich performed the first hair transplant and proved that hair from the back of the head was not susceptible to the balding process and could grow if transplanted to the frontal areas of the scalp. This is true for men and women.

Since this time, the art of hair transplantation has flourished with several doctors and clinics performing this procedure. Several patients including myself have stated that a hair transplant has changed their life. Adding hair back creates that very important frame for the face and gives you a more youthful appearance.

Hair transplantation in African Americans is a very successful procedure. In both men and women the success can exceed 90\%. Many hair loss suffers and clinicians are not aware of this simple fact. Most of the advertising for hair transplantation is not aimed at our community giving the impression the procedure must be for others. This is the furthest from the truth.African American hair is naturally course and curly which gives the appearance of greater density when transplanted. This is the ideal situation.

A hair transplant is an outpatient procedure done under local anesthesia while you are awake, similar to a dental procedure. There are 3 main steps to the procedure. First, hair is removed from the "donor hair area" - usually the back or sides of the head. Second, openings are created on the scalp called recipient sites to place the hair follicles. Third, the grafts or hair follicles are carefully placed in position to allow for natural looking hair. A typical procedure takes about 4-6 hours. The transplanted hair does not grow immediately and usually takes 2-3 months to start growing. Usually, by one year the new hair growth is realized.Complications following a hair transplant are very rare. Occasionally ingrown hairs can be experienced as the transplanted hair follicles begin to produce a new hair. This is easily treated with warm compresses and if needed a short course of antibiotics. A hair transplant should only be performed by a skilled surgeon with extensive experience and certified by the American Board of Hair Restoration Surgery.

Below are examples of a male and female African American patient's of mine with typical results after hair transplantation.

A typical African American male in his 30's who was losing his hairline and looks 10 years older because of this. He was very selfconscience about his appearance and sought help. We decided to perform an FUE (Follicular Unit Extraction) hair transplant on him. This procedure is ideal for African Americans who like to wear their hair short. We remove one hair follicle at a time from the back of the head with a punch technique. This does not leave the typical linear scar across the back of the head that is easily seen with short hair and is present with traditional hair transplant methods (Figure 1).

Traction Alopecia is very common among African American females. The usual causes are hair styling practices such as weaving and braiding causing damage to the hair follicle. Hair transplantation is a wonderful option to restore a woman's hairline and the temporal areas of the scalp. With the usual traction alopecia patient, it might take more than one transplant to achieve the desired results. When performing this procedure on an African American female, I usually collaborate with an experienced dermatologist to achieve the optimal results for

Correspondence to: William D Yates, Yates Hair Science Group, 565 Lakeview Parkway, Suite 108, Vernon Hills, IL 60061, 100 E Walton, Suite 400, Chicago, IL, USA, Tel: 312-2610872; Fax: 224-206-7443; E-mail: yates7579@yahoo.com

Received: August 01, 2015; Accepted: August 24, 2015; Published: August 26, 2015 


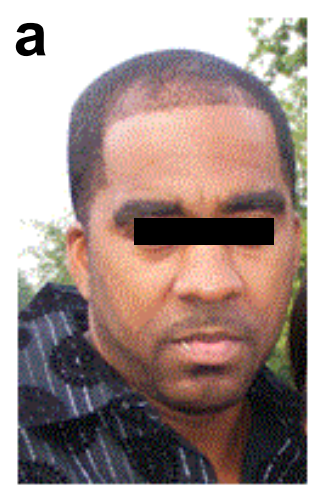

b

Figure 1. 900 grafts; 1 year post-op

Patient E.B. "The only regret that I have is that I didn't have a transplant sooner. I look so much better and younger and my confidence has soared. The best thing is I can wear my hair short in the back."
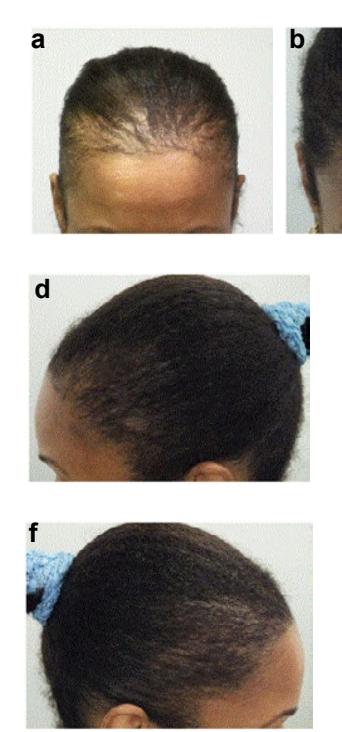

Figure 2. 1652 grafts; 9 months post op.
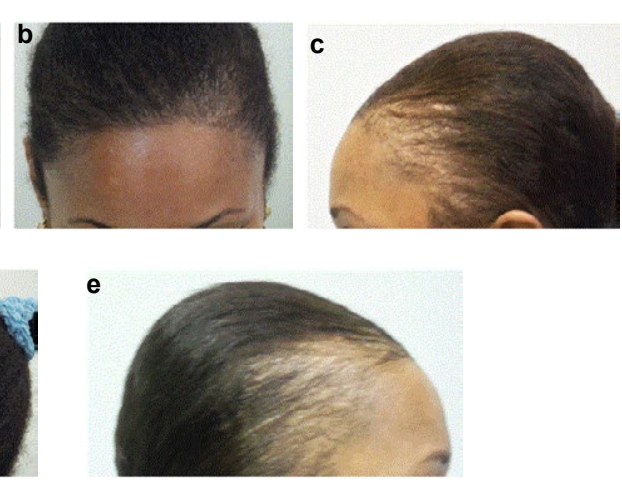

. these patients. Following are photos of typical case. A healthy female who wore braids for many years. She was determined an excellent candidate and hair transplantation was performed (Figure 2).

I would recommend the following for anyone suffering from hair loss

1) Seek the opinion of a dermatologist experienced in treating hair loss. Ask if finasteride or minoxidil is appropriate for your hair loss.

2) Eat a healthy diet and exercise regularly

3) If your hair loss is from chemicals or any hair styling practices, stop them immediately. Choose other hair styles.

Make an appointment with a board certified hair transplant surgeon that is familiar with your type of hair and hair loss if you are interested in hair transplantation.

\section{References}

1. http://www.statisticbrain.com/hair-loss-statistics/ 